\title{
Implementation and Validation of Range Imaging on a UHF Radar Wind Profiler
}

\author{
PHILLIP B. CHILSON \\ Cooperative Institute for Research in Environmental Sciences, University of Colorado, and \\ NOAA/Environmental Technology Laboratory, Boulder, Colorado \\ TIAN-YOU Yu \\ National Center for Atmospheric Research, Boulder, Colorado \\ Richard G. Strauch AND ANDREas Muschinski \\ Cooperative Institute for Research in Environmental Sciences, University of Colorado, and \\ NOAA/Environmental Technology Laboratory, Boulder, Colorado \\ ROBERT D. PALMER \\ Department of Electrical Engineering and Center for Electro-Optics, University of Nebraska at Lincoln, Lincoln, Nebraska
}

(Manuscript received 19 September 2002, in final form 23 December 2002)

ABSTRACT

\begin{abstract}
The available range resolution of pulsed radar wind profilers is usually limited by bandwidth restrictions. Range imaging (RIM) has recently been developed as a means of mitigating these limitations by operating the wind profilers over a small set of distinct transmitter frequencies. A constrained optimization method can then be used to generate high-resolution maps of the reflectivity field as a function of range. This paper presents a description of how the RIM technique has been recently implemented on the Platteville 915-MHz tropospheric profiler, the first such implementation at UHF. Examples of data collected during a two-part experiment on 10 April 2001 using the Platteville 915-MHz tropospheric profiler are presented. In the first part, an intercomparison was made involving measurements from RIM and standard radar techniques. It is shown that available frequency bandwidth can be very effectively utilized through the RIM processing. In the second part of the experiment, RIM was applied to radar observations collected with a short $(0.5 \mu \mathrm{s})$ transmit pulse. The resulting data include observations of a thin, persistent scattering layer attributed to a subsidence inversion and billows from a KelvinHelmholtz instability. Estimates of the width of the layer were found to be as small as $12 \mathrm{~m}$.
\end{abstract}

\section{Introduction}

There are a host of phenomena associated with the atmospheric boundary layer (ABL) and lower free atmosphere that manifest themselves within narrow, wellconfined height intervals. Often, measurements of these phenomena are critical to our understanding of the structure and evolution of the ABL. This in turn impacts our understanding of the vertical exchange of mass and momentum, as well as the generation of turbulence. Examples of these are the height and thickness of the entrainment zone, internal gravity waves in stably stratified flows, and Kelvin-Helmholtz instabilites.

Observations have been made of persistent layers in the atmosphere attributed to both turbulence and sharp

Corresponding author address: Dr. Phillip B. Chilson, NOAA/ Environmental Technology Lab, 325 Broadway, R/ET2, Boulder, CO 80305-3328.

E-mail: phillip.chilson@noaa.gov gradients in temperature and humidity (Dalaudier et al. 1994; Muschinski and Wode 1998; Luce et al. 2001a; Worthington 2001). In addition to being intriguing from a dynamical point of view, there is also interest in better understanding how such layers impact on radar backscatter (Luce et al. 1995; Muschinski 1997; Muschinski and Wode 1998; Chilson et al. 2001). Finally, there are numerous reports pertaining to the relevance and observation of gravity waves in stably stratified flows in or near the ABL (e.g., Gossard et al. 1970; Gossard 1990; Eaton et al. 1995). Often the features of these waves, such as amplitude, period, and thickness of the underlying layers, cannot be satisfactorily resolved with conventional boundary layer profilers that provide height resolutions of 60-100 m (Angevine et al. 1993).

Keeping the above discussion in mind, it is not surprising that many different approaches have been explored to obtain high-resolution observations of the atmosphere using radar wind profilers (e.g., Hocking 
1997). One method is to operate the wind profilers using very short pulses, which translates into better range resolution (Rüster et al. 1998). However, bandwidth restrictions are often a limiting factor. Furthermore, radars operating with shorter pulses suffer losses in signal-tonoise ratio (SNR) unless pulse compression schemes are employed (Schmidt et al. 1979). Range resolution on the order of $1 \mathrm{~m}$ can be obtained through measurements with frequency-modulated continuous-wave (FMCW) radars (Richter 1969). However, FMCW radars (commonly operated at a $10-\mathrm{cm}$ wavelength) are hampered by limitations that preclude their use for operational measurements, such as restricted altitude coverage (due to the wavelength), sensitivity to biological targets, and difficulties in measuring Doppler velocities with good range resolution.

Several approaches that involve the use of closely spaced radar frequencies have been advanced to mitigate bandwidth limitations of pulsed radars. One of the these is frequency-domain interferometry (FDI), which exploits the interference pattern between the coherently detected signals from the two frequencies (e.g., Kudeki and Stitt 1987; Franke 1990). In FDI there exists an underlying assumption that the reflectivity structure within the resolution volume possesses a single horizontal Gaussian-shaped layer.

The assumption imposed by FDI are often not satisfied in the atmosphere. Franke (1990) proposed the use of more than two transmitter frequencies as an extension to FDI; however, no experimental or simulated data were presented to verify the theory. This initial multiple-frequency work has recently been advanced through the use of advanced optimization techniques, which in this paper are collectively referred to as range imaging (RIM) (Palmer et al. 1999; Luce et al. 2001b). Simulations using RIM clearly illustrate that RIM could be used to overcome the single-layer assumption of the FDI method and provide an estimate of the reflectivity structure along the radial dimension within the resolution volume (Palmer et al. 1999).

The paper is divided into two main parts. We begin by giving a short introduction to RIM and how it was implemented on the Platteville $915-\mathrm{MHz}$ tropospheric profiler. Next, we present the objectives and realization of a two-part RIM experiment that was conducted on the radar. Finally, results of the experiment are shown and discussed.

\section{Range imaging}

RIM was developed in an attempt to expand the idea of FDI to the general case of $m$ different transmitter frequencies (Palmer et al. 1999; Luce et al. 2001b). After coherent integration, subject to the same constraints as temporal sampling, the $m$ coherently detected signals will be denoted by $s_{1}(t), s_{2}(t), \ldots, s_{m}(t)$. For notational convenience, the $m$ signals are combined into a column vector $\mathbf{s}(t)$, providing a more compact nota-

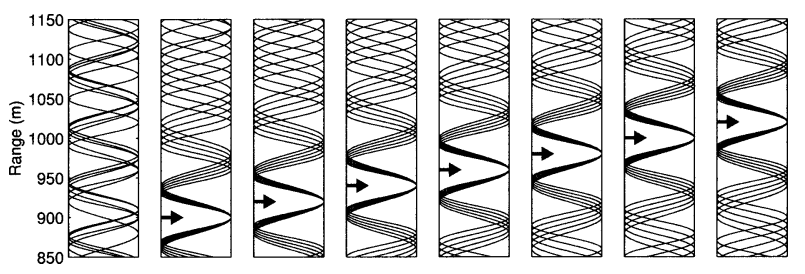

FIG. 1. Illustrative example of the effect of the range-steering vector on the $m$ signals used in the RIM procedure. The left-most panel shows the $m$ signals without phase synchronization, while the remaining panels provide examples of the use of the range-steering vector for different values of $\tilde{r}$. The respective values of $\tilde{r}$ are denoted by the thick arrow. Note that the wavelengths of the carrier signals shown have been exaggerated for the sake of demonstration.

tion. The ultimate question is how to use the frequency diversity, or bandwidth, that exists within the signals spanned by $\mathbf{s}(t)$. Arguably, the simplest composite signal that can be conceived is the weighted summation of the $m$ signals, which is given in matrix notation as $\mathbf{y}(t)=$ $\mathbf{w}^{\dagger} \mathbf{s}(t)$, where the dagger represents the Hermitian operator (complex transpose). The complex weights $w_{1}$, $w_{2}, \ldots, w_{m}$, corresponding to each of the coherently detected signals, are combined in the column vector $\mathbf{w}$. Note that the weights are complex, thus providing the flexibility of both amplitude and phase modification. Basically, the weight vector will be used to create constructive interference between the $m$ signals at a particular range $(\tilde{r})$ within the normal range-resolution volume. This concept is illustrated in Fig. 1.

Various inversion techniques used to obtain estimates of the range brightness (range power distribution) can be found in Palmer et al. (1999) and Luce et al. (2001b). The simplest of these is FDI. The FDI technique together with some of its limitations has already been discussed in the introduction. The maximum entropy method (MEM) can be used to process datasets that include more than one carrier frequency with good results. We note that MEM places high demands upon computer resources and is not significantly better than the Capon method (discussed below) for data with high SNR (Yu and Palmer 2001). Singular value decomposition methods such as the multiple signal classification (MUSIC) algorithm are discussed in Luce et al. (2001b) and Smaïni et al. (2002). These methods can be very effective for determining the height of very thin atmospheric scattering layers, but not for determining their intensities or widths. Finally Smaïni et al. (2002) discuss the application of the Lagunas-Gasull inversion method in connection with wind profiler data. This method is similar to the Capon method (Stoica and Moses 1997).

In the present study, we focus on the estimation of the range-power distribution using the Fourier and $\mathrm{Ca}-$ pon methods (Palmer et al. 1999; Luce et al. 2001b). The essential distinction between the two is that the Capon method is adaptive, whereas the Fourier method is not. That is, the Capon method uses a constrained optimization procedure. An immediate advantage of 
adaptive processing is that interference and range sidelobe effects can be suppressed by judiciously choosing the weighting vector $\mathbf{w}$ based on the data being processed (Palmer et al. 1999; Luce et al. 2001b).

\section{RIM implementation on the Platteville 915-MHz tropospheric profiler}

The Platteville 915-MHz tropospheric profiler was built in 1983 and was operated as a wind/radio acoustic sounding system (RASS) profiler at Denver, Colorado's, Stapleton International Airport from 1983-95. It was part of the Colorado network (Strauch et al. 1984) and led to the development of the National Oceanic and Atmospheric Administration's (NOAA) 30-station UHF wind profiler network that was installed in the late 1980s and early 1990s (Weber et al. 1990). The 915-MHz radar was moved in 1997 from Stapleton International Airport to its current location at the Platteville Atmospheric Observatory $\left(40.19^{\circ} \mathrm{N}, 104.73^{\circ} \mathrm{W}, 1521 \mathrm{~m}\right.$ above mean sea level) in Platteville, Colorado. The Platteville Atmospheric Observatory is operated jointly by the Environmental Technology Laboratory and Aeronomy Laboratory, both of NOAA, and the Cooperative Institute for Research in Environmental Sciences (CIRES) at the University of Colorado.

The transmitted pulse is nominally generated by mixing a pulsed sample of the $945-\mathrm{MHz}$ local oscillator (LO) with a pulsed sample of the $30-\mathrm{MHz}$ coherent oscillator (COHO). For the current RIM implementation, the LO frequency is changed just prior to the generation of each transmitted pulse so that every $n$th transmitted pulse has the same frequency, where $n$ is the number of frequencies, typically four in our applications. Changing the $\mathrm{LO}$ rather than the $\mathrm{COHO}$ means that the allowable frequency shift is not limited by relatively narrowband intermediate frequency (IF) components but by broader-bandwidth radio frequency (RF) components.

The radar data system used the profiler online program (POP) developed by Carter et al. (1995) that can support multiplexed inputs from multiple receivers that are used in spaced antenna (SA) systems and interferometers. For our RIM experiments, the input signals are from a single receiver; the data system treats the incoming frequency multiplexed data stream exactly as it would treat inputs from multiplexed separate receivers. Signal samples for each range gate are separated, and a number of complex video samples from the same transmitted frequency are summed. The versatility of the POP system allowed us to conduct RIM experiments with no changes to the data system or software. After time-domain integration, the digitized complex video time series for each transmitted frequency and each range gate were stored for offline analysis. Following the acquisition of a time series segment, there is a gap in the time series records while POP computed a fast
TABLE 1. Operational parameters during the experiments. Measurements are made using four frequencies.

\begin{tabular}{|c|c|c|c|}
\hline \multirow[b]{2}{*}{ Characteristic } & \multicolumn{3}{|c|}{ Value } \\
\hline & $\begin{array}{l}\text { 300-m RIM } \\
\text { mode }\end{array}$ & $\begin{array}{l}\text { 75-m } \\
\text { standard } \\
\text { mode }\end{array}$ & $\begin{array}{l}\text { 75-m RIM } \\
\text { mode }\end{array}$ \\
\hline \multicolumn{4}{|l|}{ Fundamental parameters } \\
\hline Pulse width (ms) & 2 & 0.5 & 0.5 \\
\hline Interpulse period (ms) & 67 & 67 & 41 \\
\hline Sampling period (ms) & 1 & 0.5 & 0.5 \\
\hline Time domain averaging & 60 & 60 & 90 \\
\hline Number of spectral points & 256 & 128 & 256 \\
\hline Number of repetitions & 5 & 1 & 1 \\
\hline Spectral averaging & 1 & 8 & 1 \\
\hline \multicolumn{4}{|l|}{ Resulting parameters } \\
\hline Nyquist velocity $\left(\mathrm{m} \mathrm{s}^{-1}\right.$ ) & 5.1 & 5.1 & 5.6 \\
\hline Dwell (s) & 4.1 & 16.5 & 3.8 \\
\hline Time per repetition (s) & 6.2 & 18.4 & 6.6 \\
\hline Time per mode $(\mathrm{s})$ & 31 & 18.4 & 6.6 \\
\hline \multicolumn{4}{|l|}{ Height sampling } \\
\hline First height (m) & 480 & 1080 & 405 \\
\hline Height resolution (m) & 300 & 75 & 75 \\
\hline Height spacing (m) & 150 & 75 & 75 \\
\hline Number of heights & 36 & 28 & 36 \\
\hline
\end{tabular}

Fourier transform (FFT) and wrote the time series and FFT data to disk.

\section{Experimental setup and data processing}

On 10 April 2001, a two-part experiment was conducted. The first part was designed to demonstrate and validate the application of RIM on a UHF profiler for studies of the boundary layer and lower free troposphere. In this portion of the experiment, a self-consistency check was performed by operating the radar alternately between RIM and conventional modes. RIM measurements were made at 300-m resolution (300-m RIM mode) and then compared against the conventional measurements made at $75-\mathrm{m}$ resolution $(75-\mathrm{m}$ standard mode). Throughout the text, we refer to the method of using moment calculations from a single-frequency Doppler autospectrum to derive estimates of the backscattered power, radial velocity, and the variance of the radial velocity as conventional processing (e.g., Woodman 1985). In the second part of the experiment, observations were made in the RIM mode with a $75-\mathrm{m}$ range resolution (75-m RIM mode). A listing of the operational parameters for the experiment are given in Table 1. Further particulars of the experiment are discussed below.

In the first part, the radar was operated for $2 \mathrm{~h}$ at the frequencies of 914, 914.33, 915.33, and $916 \mathrm{MHz}$ alternatingly in the 300-m RIM and 75-m standard modes (see Table 1). The choice of a nonuniform frequency spacing reduces the redundancy in the available frequency lags (Palmer et al. 2001). RIM analyses were performed on the data obtained while operating in the 


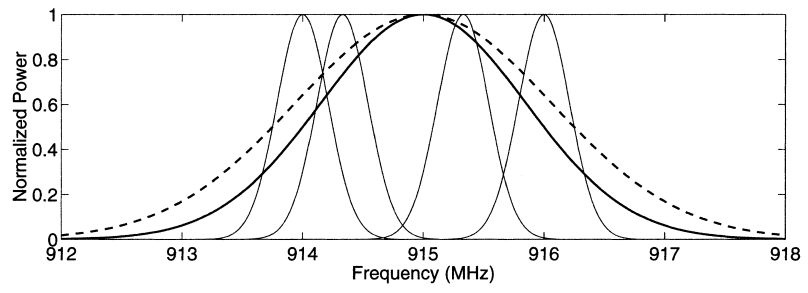

FIG. 2. Power spectra of the transmit pulses for the 300-m RIM mode (light solid lines) and the 75-m standard mode (heavy solid line). Gaussian-shaped transmit pulses have been assumed. Also shown (heavy dashed line) is power spectrum for a $0.4-\mu$ s pulse (requiring $2.5 \mathrm{MHz}$ of bandwidth).

first mode. Data resulting from the second mode were subject to conventional processing (e.g., Carter et al. 1995). The 75-m range resolution provided a reference by which the RIM results could be compared. Although four frequencies were transmitted and received in the 75-m standard mode, conventional processing was executed on the four data streams separately and then averaged.

With this particular radar system, the available bandwidth can be more effectively exploited using RIM than through the transmission of shorter pulses. The bandwidth of the power amplifier for the Platteville 915$\mathrm{MHz}$ tropospheric profiler is more than $10 \mathrm{MHz}$. It should, therefore, be possible to amplify pulses shorter than $0.5 \mu \mathrm{s}$. However, as was common with solid-state power amplifiers built in the early 1980s, it cannot be operated with fast rise or fall times without risk of damage from transients. Therefore, it can operate over a relatively wide frequency range with pulse durations of $0.5 \mu \mathrm{s}$ or more and is, therefore, ideal for RIM experiments. Also, pulse compression with binary phase codes, commonly used with wind profilers, is also restricted to code-chip durations of $0.5 \mu$ s or more for the same reason. Pulse compression with a broadband chirp pulse would be possible, but is not built into the system.

Figure 2 has been created to illustrate the difference between how bandwidth is occupied in the 300-m RIM and $75-\mathrm{m}$ standard modes. The $0.5-\mu$ s pulse in the $75-$ $\mathrm{m}$ standard mode requires $2 \mathrm{MHz}$ of bandwidth. The frequency spacing for the 300-m RIM mode required $2.5 \mathrm{MHz}$ of bandwidth ( $2 \mathrm{MHz}$ for the actual frequency spread and $500 \mathrm{kHz}$ for the $2-\mu$ s pulse). The bandwidth occupied in the 300-m RIM mode has been chosen to closely match that for the 75-m standard mode for the sake of comparison. We have also considered the power spectrum for a $0.4-\mu$ s transmit pulse. Such a pulse requires the same amount of bandwidth as used in the 300-m RIM mode. Note that the tails of the power spectra for the 0.4- and $0.5-\mu$ s transmit pulses are considerably wider than those for the RIM mode. An immediate advantage of using the RIM approach is the additional transmitted power afforded through the longer pulses, which translates into an increase in SNR.
The second part of the experiment was conducted in the 75-m RIM mode for $1 \mathrm{~h}$ and at the frequencies of 913, 913.67, 915.67, and 917 MHz. The wider separation of frequencies compared with the 300-m RIM mode was chosen in conjunction with the selection of a shorter transmitted pulse width. In this way, it was hoped to achieve even better resolving power through RIM processing. Whereas the time series data were oversampled in the 300-m RIM mode (see Table 1), there was no oversampling in the 75-m RIM mode. Due to limitations in processor memory, it was only possible to achieve the desired height coverage of the radar measurements by not oversampling.

\section{Results}

All data collected during the experiment were processed using three different methods. These are the Fourier and Capon techniques as described in Palmer et al. (1999) and Luce et al. (2001b), and conventional processing (e.g., Woodman 1985). An overview of the processed data from the 300- and 75-m RIM modes for the entire experiment is given in Fig. 3 as range-time pseudocolor (RTP) plots. Data from the 75-m standard mode are not shown. This plot, as in all RTP plots, has not been range corrected. Furthermore, we note that the difference between local time during the observations and universal time is $6 \mathrm{~h}$. That is, the data shown in Fig. 3 correspond to 0900-1200 local time.

In the Fourier and Capon techniques, one is free to choose the number of values for $\tilde{r}$ to be assigned within a nominal range gate. We refer to the resulting subdivisions of the nominal range gate as subgates. Note that the choice for the width of the subgate does not determine the resolution that can be achieved with RIM. For the current data analysis, 50 values of $\tilde{r}$ were chosen for each nominal range gate. Therefore, the resulting widths of the subgates for the 300- and 75-m RIM modes are 6 and $1.5 \mathrm{~m}$, respectively.

There are obvious differences as well as similarities between the RTP plots shown in Fig. 3. Each RTP plot reveals a persistent and well-defined layer at a height of about $1.35 \mathrm{~km}$ and a braided structure occurring between 1730 and 1800 UTC and at heights between 1.5 and $2.0 \mathrm{~km}$. The layer and the braided structure most likely resulted from a subsidence inversion and a Kelvin-Helmholtz instability, respectively. The layer of enhanced signal strengths occurring at a height of 900 $\mathrm{m}$ is due to interference. The difference in the noise levels (background color) during the first and second parts of the experiment result from the different bandwidths of the filters used. A narrower filter was used for the 300-m RIM mode than was used for the $75-\mathrm{m}$ RIM mode. Furthermore, the shorter transmit pulse widths used for the second part of the experiment is responsible for a reduction in signal power.

Figure 3 illustrates several fundamental ideas behind the use of imaging techniques to improve resolution. 

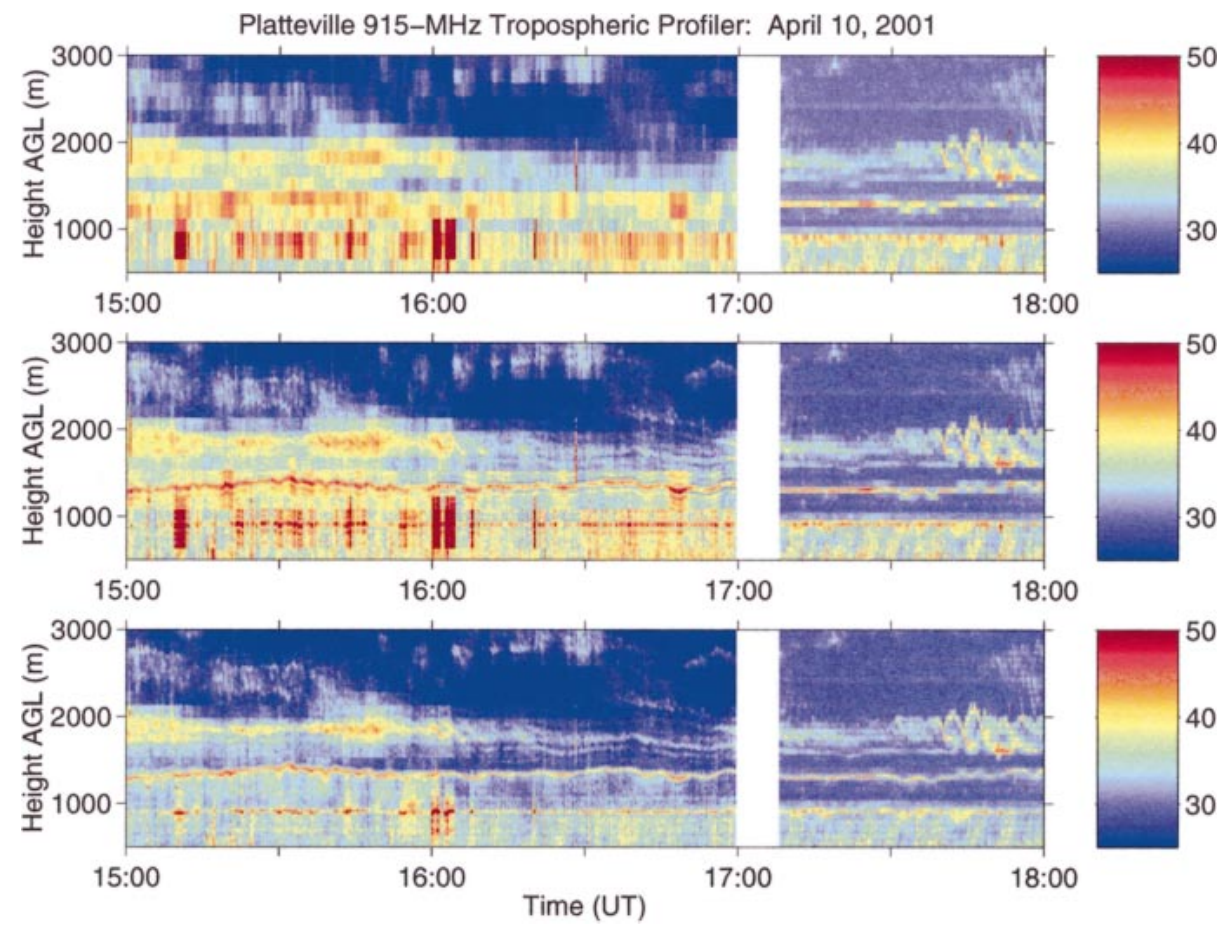

FIG. 3. RTP plots of the (top) backscattered power and (middle and bottom) brightness expressed in decibels. Relatives units are shown. Estimates of the brightness were obtained using the (middle) Fourier and (bottom) Capon methods. The color scale ranges from 25 to $50 \mathrm{~dB}$. See text for additional information.

An obvious difference between results from the Fourier and Capon RIM processing, and one that has been discussed in numerous publications (e.g., Palmer et al. 1999; Luce et al. 2001b; Palmer et al. 2001), is the image contamination by "ghost layers." By ghost layers we mean artificial enhancements in the brightnesses resulting from range sidelobes in the Fourier rangeweighting pattern. We further note that the Capon RIM processing has produced narrower estimates of the layer's thickness than those from Fourier processing. This is to be expected for observations of a thin, well-defined layer and has been demonstrated in simulations (Palmer et al. 1999; Luce et al. 2001b).

\section{a. 300-m RIM self test}

The persistent layer was observed in range gates 6 , 7 , and 8 for the 300-m RIM mode. The appearance of one layer in three consecutive gates is a result of the oversampling. These gates are centered at heights of $1.23,1.38$, and $1.53 \mathrm{~km}$, respectively. Values of the brightness are shown in Fig. 4 for the three adjacent gates over a 2-h period. The center height of each gate is indicated by a black line. Results from the Fourier RIM are not shown because of the degradation of image quality caused by shadow images. Consistency in the layer position, width, and intensity can be clearly observed in Fig. 4.

In order to further verify RIM results quantitatively, the layer position for the three gates shown in Fig. 4 have been estimated and are given in the upper panel of Fig. 5 . The estimates were calculated by simply finding those heights corresponding to peak values of the range brightness profiles for each range gate. Only heights for brightness values above a certain threshold are shown. Additionally, the second central moment of the range brightness for range gate 7 was calculated. The layer thickness, defined as twice the square root of the second central moment, are shown in the lower panel of Fig. 5. The mean, median, and standard deviation of the layer thickness over the 2-h period were 30,29 , and $8 \mathrm{~m}$, respectively. The minimum layer thickness was $16 \mathrm{~m}$.

\section{b. 300- and 75-m RIM intercomparison}

Because there are no supporting in situ measurements for these radar observations, we use the 75-m standard mode data for comparison with the 300-m RIM results. As previously mentioned, the two modes were interleaved, and the time intervals dedicated to each mode are given in Table 1. We take as a working hypothesis that the radar data collected from the two modes can be treated as quasi-independent observations. To facilitate the comparison between the two modes, the subgate spacing for the RIM analysis has been set to $75 \mathrm{~m}$ to correspond with the range resolution of the $75-\mathrm{m}$ standard mode data. Figure 6 provides a qualitative comparison of the results from the 300-m RIM and 75-m 


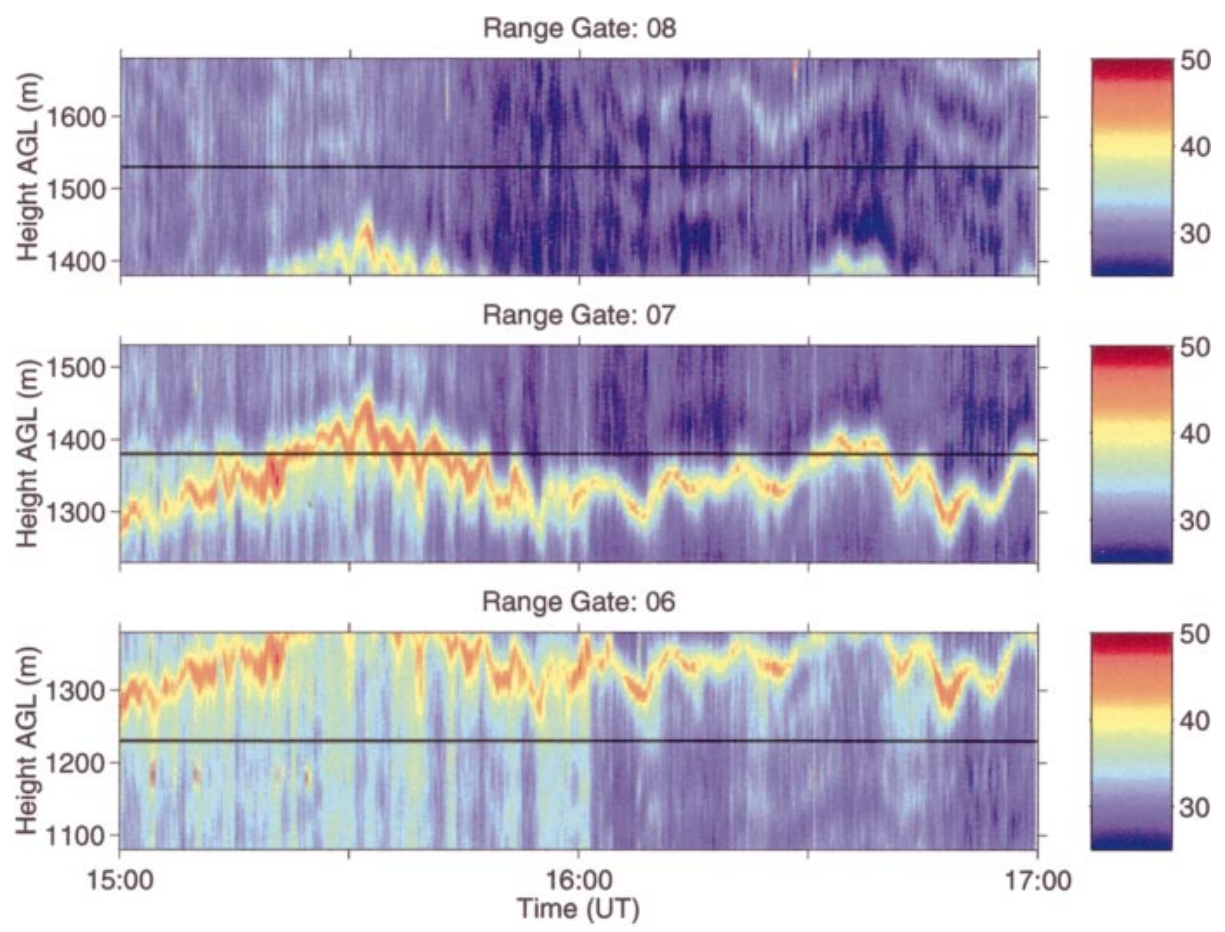

FIG. 4. The Capon range brightness from gates 6, 7, and 8 . The center of each gate is indicated by a solid line. Note that a $50 \%$ oversampling in range was implemented in this experiment. It is evident that the layer in the lower half of gate 7 is consistent with the layer in the upper half of gate 6 . Similar correlation can be observed between gates 7 and 8 as well. The result demonstrates that RIM produces self-consistent estimates from gate to gate. The color scale ranges from 25 to $50 \mathrm{~dB}$.

standard modes. As already mentioned in reference to Fig. 3, differences in the noise floor and signal levels are expected between the 75-m standard and 300-m RIM modes. These result from the differences in the transmit pulses and filter bandwidths are used for the two modes.

Examples of single height profiles of the range bright-
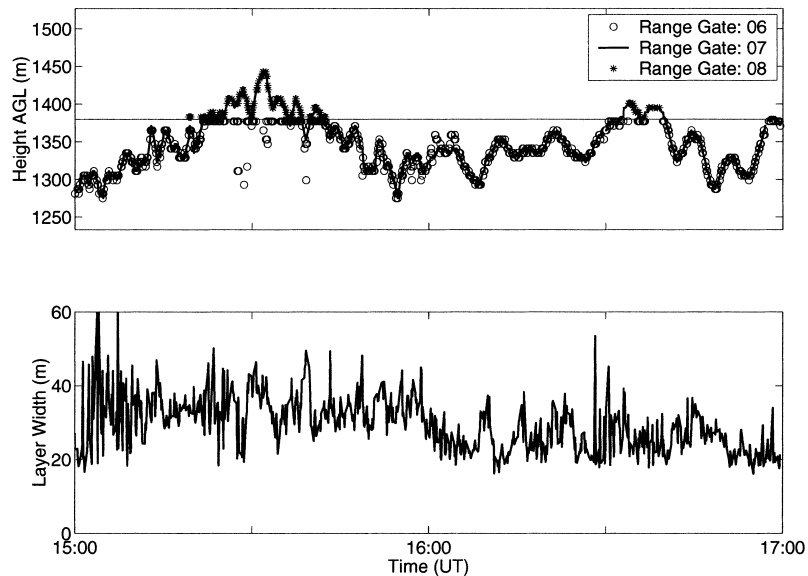

FIG. 5. (top) A comparison of layer position estimated using the Capon range brightness as shown in Fig. 4. The layer positions estimated from the three adjacent gates were consistent except when the layer observed at gate 6 moved toward the upper boundary. (bottom) Estimate of the layer width calculated using data from range gate 7 . nesses and backscattered power taken from Fig. 6 are presented in Fig. 7. Each profile was constructed from a single dwell recorded at 1500 UTC. Because the data collected in $75-\mathrm{m}$ standard mode are from a different dwell than those for the RIM-processed data, there is a time offset of about $30 \mathrm{~s}$ between the profiles. Here again, differences in the profiles between the 75-m standard mode and the RIM modes can be partly attributed to the differences in the transmit pulses and filter bandwidths.

We now focus again on that height at which the persistent subsidence layer was observed. Measurements from four range gates in the $75-\mathrm{m}$ standard mode coincide in height with measurements taken from a single range gate in the 300-m RIM mode (300-m resolution). In Fig. 8, we present RTP plots of the brightness estimates calculated using both the Fourier and Capon RIM methods and an RTP plot of the backscattered power calculated for the corresponding range gates in the 75$\mathrm{m}$ standard mode. For this comparison, 50 subgates were chosen; therefore, the subgate width is $6 \mathrm{~m}$.

An interesting result that can be inferred from Fig. 8 is a qualitative comparison of how effectively radar bandwidth has been used in the different modes and processing procedures. The amount of bandwidth occupied in the two modes is similar. The frequency spacing for the 300-m RIM mode required $2.5 \mathrm{MHz}$ of bandwidth, which could have been used to obtain a range 


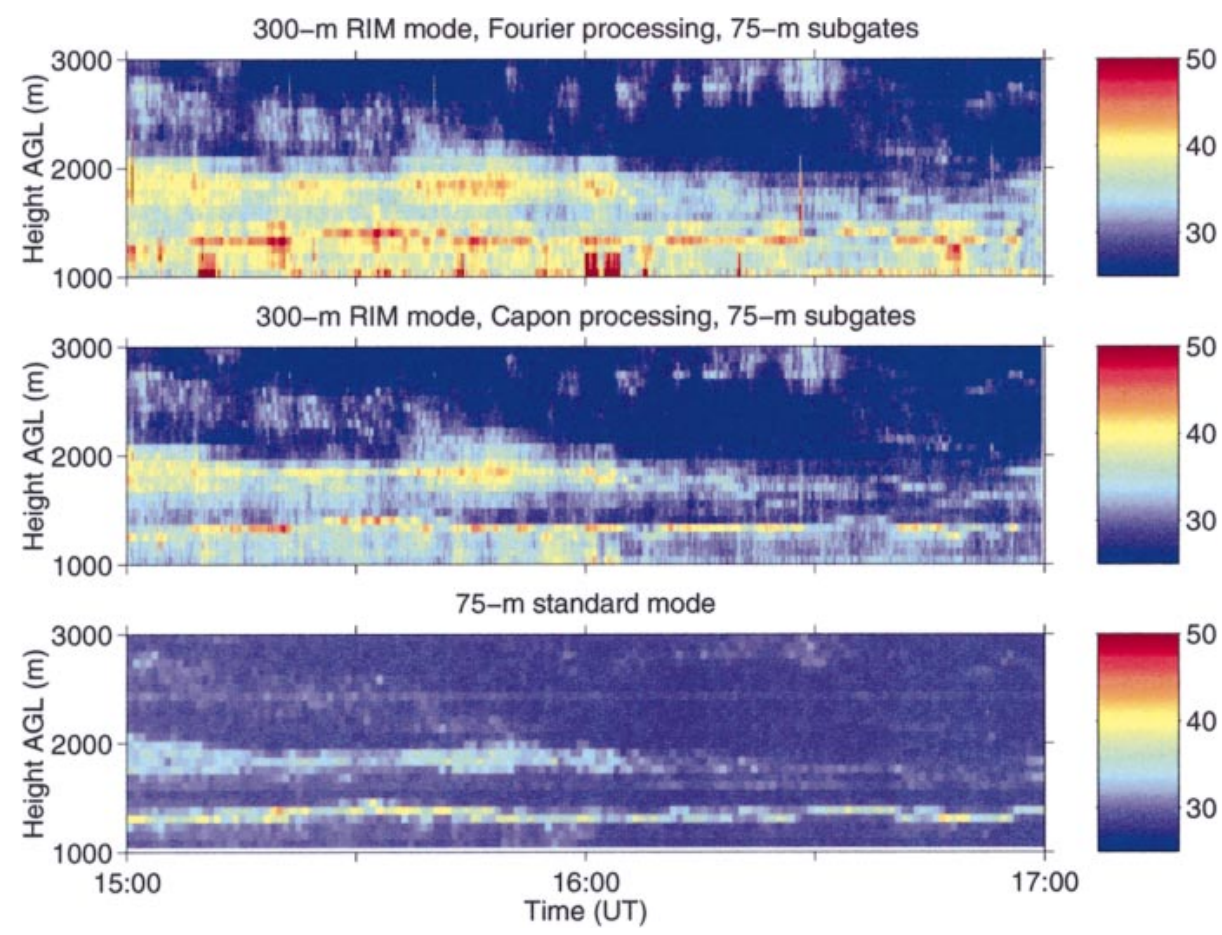

FIG. 6. RTP plots of the (top and middle) brightness and (bottom) backscattered power expressed in decibels. Estimates of the brightness were obtained using the (top) Fourier and (middle) Capon methods. The color scale ranges from 25 to $50 \mathrm{~dB}$.

gate spacing of $60 \mathrm{~m}$ if the bandwidth were used to transmit a narrower pulse. We can refer to this as the equivalent range resolution for a given bandwidth. However, results from the Capon RIM analysis show the thin persistent layer to have an average width of $30 \mathrm{~m}$. A bandwidth of $5 \mathrm{MHz}$ would be needed to obtain an equivalent range gate spacing of $30 \mathrm{~m}$ using conventional processing. That is, for a given bandwidth, the Capon RIM technique is capable of producing estimates of the layer thickness that are smaller than the equivalent range resolution.

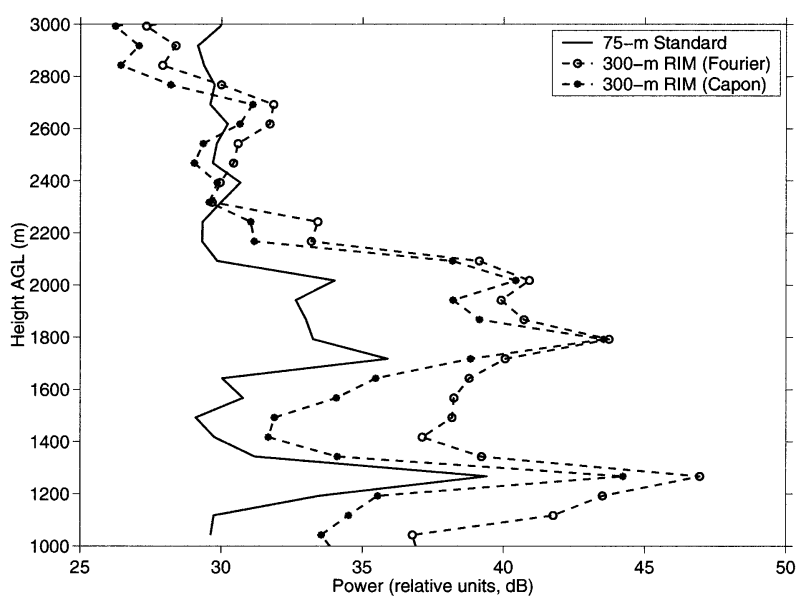

FIG. 7. Height profiles of the range brightness and backscattered power shown in Fig. 6. The profiles were taken at 1500 UTC.

\section{c. 75-m RIM}

In this section, we discuss the application of imaging techniques to the 75-m RIM mode data. The frequency spacing for the mode required $6 \mathrm{MHz}$ of bandwidth, which corresponds to an equivalent range resolution of $25 \mathrm{~m}$. In Fig. 9 a range brightness RTP plot of the brightness estimates resulting from a Capon RIM analysis is shown in the upper panel. This figure shows an enlargement of data already presented in the lower panel of Fig. 3. In Fig. 9 we have focused on the time and height corresponding to the persistent layer and the braided structure. We repeat that oversampling was not implemented in this mode. Therefore, removing the range-weighting pattern is difficult, because the process would require multiplying by the inverse of the rangeweighting pattern at heights where SNR is low. This is the cause of the dark horizontal stripes that run through the image.

RTP plots similar to the one shown in the upper panel of Fig. 9 have often been observed by FMCW radars, which have range resolutions on the order of $1 \mathrm{~m}$ (Gossard et al. 1970; Gossard 1990; Eaton et al. 1995). Indications of braided structures are also apparent in the brightness data from the first part of the experiment (see Fig. 3), but they are more difficult to resolve. The large billow structures seen in Fig. 9 are also apparent in the RTP plots of power from the conventional processing, but with less clarity. However, the undulations of the 

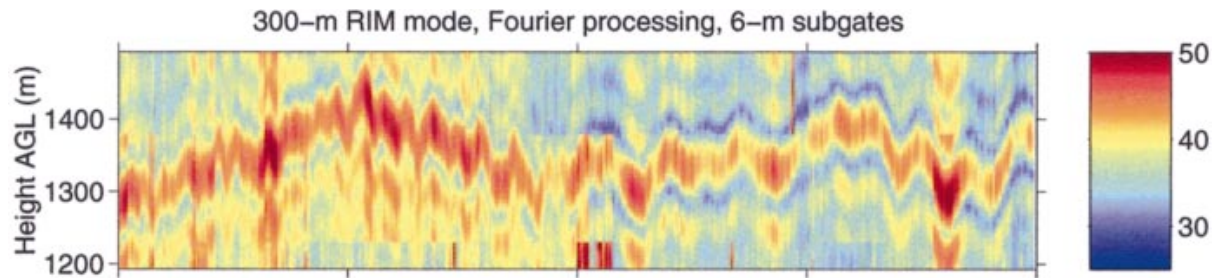

300-m RIM mode, Capon processing, 6-m subgates
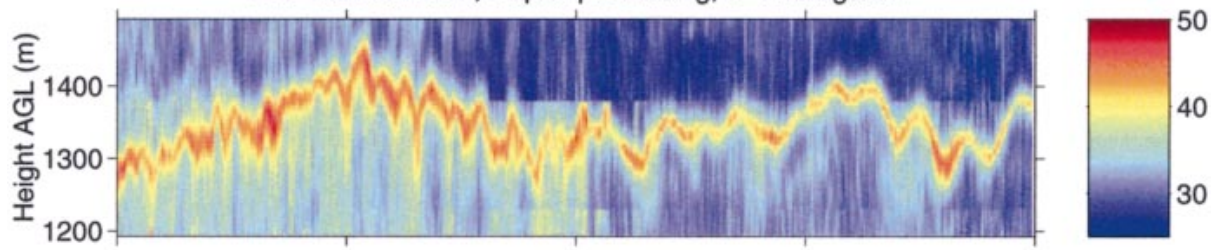

75-m standard mode

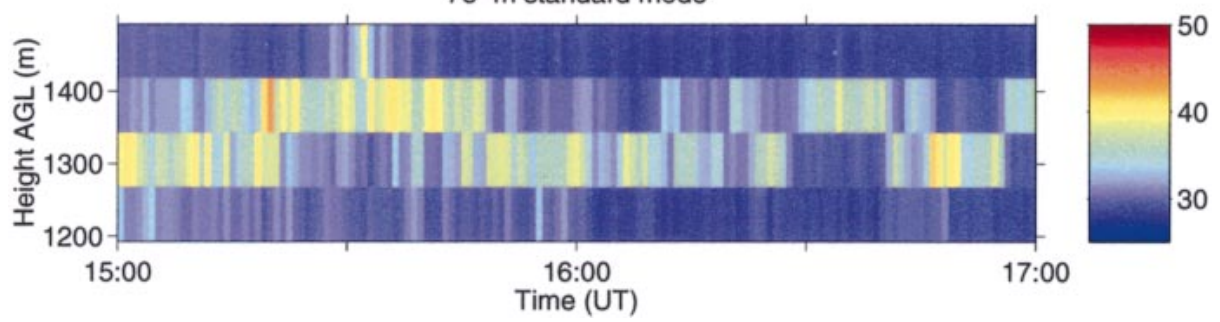

FIG. 8. RTP plots of the (top and middle) brightness and (bottom) backscattered power expressed in decibels. Estimates of the brightness were obtained using the (top) Fourier and (middle) Capon methods. The color scale ranges from 25 to $50 \mathrm{~dB}$.
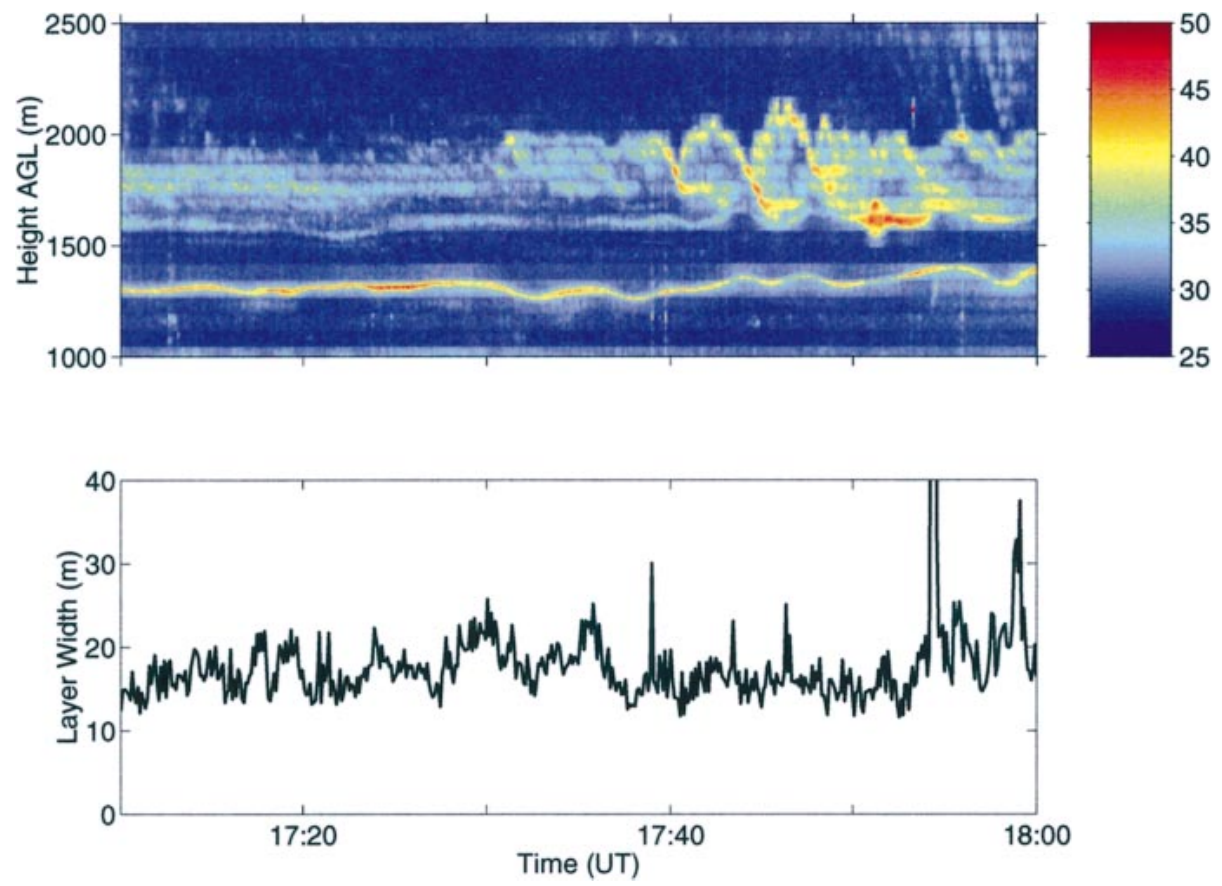

FIG. 9. (top) RTP plot of the brightness expressed in decibels. Estimates of the brightness were obtained using the Capon method. The color scale ranges from 25 to $50 \mathrm{~dB}$. (bottom) Estimate of the width of the inversion layer located at a height of $1.35 \mathrm{~km}$. 
layer structure at $1.35 \mathrm{~km}$ are mostly masked in the conventionally processed data.

Also shown in Fig. 9 are estimates of the width of the layer seen at a height of $1.35 \mathrm{~km}$. Similar to the data shown in Fig. 5, the layer thickness is defined as twice the square root of second central moment of the range brightness. The mean, median, and standard deviation of the layer thickness over the 1-h period were 18,17 , and $6 \mathrm{~m}$, respectively. The minimum layer thickness was $12 \mathrm{~m}$. These values are about $1 / 3$ smaller than those obtained for the same layer using the 300-m RIM mode. We must take into consideration, however, that the 300-m RIM and 75-m RIM modes did not run concurrently.

\section{Conclusions}

Range imaging (RIM) has recently been developed as a technique for high range-resolution measurements with pulsed radars using a small set of distinct transmitter frequencies. This paper describes the implementation of RIM on the Platteville 915-MHz tropospheric profiler, the first such implementation at UHF. For this radar, RIM offers a more effective use of bandwidth than is possible through the transmission of shorter pulses. When used with constrained optimization techniques, such as the Capon method, RIM could conceivably be used as an alternative to pulse compression with binary codes.

Various inversion techniques have been proposed to obtain estimates of the range brightness. For this study we have chosen to use the Fourier and Capon methods. The Fourier method was chosen because it is relatively simple to implement and interpret the processing results. That is, the Fourier method was considered as a reference, by which to compare the results from the Capon method. The Capon method offers a good compromise between processing time and quality of performance.

By interleaving the 300-m RIM and 75-m standard modes and choosing to oversample the data from the 300-m RIM mode, we were able to intercompare results from quasi-independent data sources. High-resolution RIM data were shown to be spatially and temporally consistent through the use of the distinct layer at 1.35 $\mathrm{km}$ as a reference. The layer structure revealed by Capon RIM was shown to be qualitatively consistent in position, thickness, and intensity in three adjacent gates.

We note at this point that previous RIM observations have reported in the literature been conducted at VHF. Although the mechanisms for atmospheric scatter at VHF and UHF are similar, some differences are expected. For example, VHF observations of layered structures are often aspect sensitive (e.g., Gage and Green 1978). That is, the amount of backscattered power received from the atmosphere depends upon the incidence angle of the radio waves. This effect is much less pronounced at UHF. Of course, the choice of frequency determines the size of atmospheric structures that will be detected for the case of Bragg scatter.

In order to compare data from the RIM mode and standard mode on a pixel-by-pixel basis, the high-resolution range brightness was further processed to produce profiles of synthesized SNR with lower range resolution of $75 \mathrm{~m}$. It was found that the estimates of both the position and echo intensity of the layer using RIM are consistent with estimates from the 75-m standard mode. Except for the expected discrepancies in the signal-to-noise ratios (due to filter widths and pulse widths), the profiles of range brightness estimates from the RIM-processed subgates are similar to the estimates of the backscattered power obtained for each 75-m range gates. That is, the features that are apparent in the RIM data subgated at $75 \mathrm{~m}$ are similar to those from the conventional $75-\mathrm{m}$ resolution data.

Through the high-resolution data provided by Capon RIM, we have obtained two estimates of the average width of the layer located at a height of $1.35 \mathrm{~km}$. When using a $2-\mu$ s transmit pulse and $2.5 \mathrm{MHz}$ of system bandwidth, the mean width was found to be $30 \mathrm{~m}$. The mean width of the same layer, albeit at a later time, estimated using a $0.5-\mu$ s transmit pulse and $6 \mathrm{MHz}$ of system bandwidth was $18 \mathrm{~m}$.

It is not possible to provide definitive numbers for the range resolution possible with Capon RIM. Because the method is adaptive, the attainable resolution depends ultimately on the state of the atmosphere. However, we can state that Capon RIM should function best whenever there are abrupt discontinuities in the refractive index field. On the other hand, if the radar reflectivity is homogeneous within the sampling volume, then the range resolution defaults back to that available with conventional processing.

The implementation of RIM on radar wind profilers offers many potential uses and benefits for studies of the boundary layer and lower free troposphere. Some of these have been highlighted in the paper. Other applications of RIM for boundary layer studies include the ability to monitor the evolution of the entrainment zone, improved capabilities to observe amplitudes and periods of atmospheric waves and Kelvin-Helmholtz billows, and even the development of better models to explain the interactions of radio waves with the atmosphere.

Acknowledgments. We thank Dave Carter for all of his assistance concerning the implementation of POP for the multiple-frequency measurements. We also thank Brooks Martner and Dan Law for their comments on the original manuscript. A. Muschinski and P. B. Chilson were partially supported through a research grant by Vaisala Inc. A. Muschinski was partially supported through a research grant by the U.S. Army Research Office. 


\section{REFERENCES}

Angevine, W. M., S. K. Avery, W. L. Ecklund, and D. A. Carter, 1993: Fluxes of heat and momentum measured with a boundarylayer wind profiler radar-radio acoustic sounding system. $J$. Appl. Meteor., 32, 73-80.

Carter, D. A., W. L. Ecklund, P. E. Johnston, A. C. Riddle, J. Wilson, and C. R. Williams, 1995: Developments in UHF lower tropospheric wind profiling at NOAA's Aeronomy Laboratory. Radio Sci., 30, 977-1001.

Chilson, P. B., R. D. Palmer, A. Muschinski, D. A. Hooper, G. Schmidt, and H. Steinhagen, 2001: SOMARE-99: A demonstrational field campaign for ultra-high resolution VHF atmospheric profiling using frequency diversity. Radio Sci., 36, 695707.

Dalaudier, F., C. Sidi, M. Crochet, and J. Vernin, 1994: Direct evidence of "sheets" in the atmospheric temperature field. J. Atmos. Sci., 51, 237-248.

Eaton, F. D., S. A. McLaughlin, and J. R. Hines, 1995: A new frequency-modulated continuous wave radar for studying planetary boundary layer morphology. Radio Sci., 30, 75-88.

Franke, S. J., 1990: Pulse compression and frequency domain interferometry with a frequency-hopped MST radar. Radio Sci., 25, $565-574$.

Gage, K. S., and J. L. Green, 1978: Evidence for specular reflection from monostatic VHF radar observations of the stratosphere. Radio Sci., 13, 991-1001.

Gossard, E. E., 1990: Radar research on the atmospheric boundary layer. Radar in Meteorology, D. Atlas, Ed., Amer. Meteor. Soc., $477-527$.

— J. H. Richter, and D. Atlas, 1970: Internal waves in the atmosphere from high-resolution radar measurements. J. Geophys. Res., 75, 3523-3536.

Hocking, W. K., 1997: Recent advances in radar instrumentation and techniques for studies of the mesosphere, stratosphere, and troposphere. Radio Sci., 32, 2241-2270.

Kudeki, E., and R. Stitt, 1987: Frequency domain interferometry: A high resolution radar technique for studies of atmospheric turbulence. Geophys. Res. Lett., 14, 198-201.

Luce, H., M. Crochet, F. Dalaudier, and C. Sidi, 1995: Interpretation of VHF ST radar echoes from in situ temperature sheet observations. Radio Sci., 30, 1003-1025.

$\_,-$, and ——, 2001a: Temperature sheets and aspect sensitive radar echoes. Ann. Geophys., 19, 899-920.

, M. Yamamoto, S. Fukao, D. Helal, and M. Crochet, 2001b: A frequency domain radar interferometric imaging (FII) technique based on high resolution methods. J. Atmos. Sol.-Terr. Phys., 63, 201-214.

Muschinski, A., 1997: Turbulence and gravity waves in the vicinity of a midtropospheric warm front: A case study using VHF echointensity measurements and radiosonde data. Radio Sci., 32, $1161-1178$.

— , and C. Wode, 1998: First in situ evidence for coexisting submeter temperature and humidity sheets in the lower free troposphere. J. Atmos. Sci., 55, 2893-2906.

Palmer, R. D., T.-Y. Yu, and P. B. Chilson, 1999: Range imaging using frequency diversity. Radio Sci., 34, 1485-1496.

_ , P. B. Chilson, A. Muschinski, G. Schmidt, T.-Y. Yu, and H. Steinhagen, 2001: SOMARE-99: Observations of tropospheric scattering layers using multiple-frequency range imaging. Radio Sci., 36, 681-693.

Richter, J. H., 1969: High resolution tropospheric radar soundings. Radio Sci., 4, 1261-1268.

Rüster, R., G. D. Nastrom, and G. Schmidt, 1998: High-resolution VHF radar measurements in the troposphere with a vertically pointing beam. J. Appl. Meteor., 37, 1522-1529.

Schmidt, G., R. Rüster, and P. Czechowsky, 1979: Complementary code and digital filtering for detection of weak VHF radar signals from the mesosphere. IEEE Trans. Geosci. Electron., GE-17, $154-161$

Smaïni, L., H. Luce, M. Crochet, and S. Fukao, 2002: An improved high-resolution processing method for a frequency domain interferometric FII technique. J. Atmos. Oceanic Technol., 19, 954-966.

Stoica, P., and R. Moses, 1997: Introduction to Spectral Analysis. Prentice Hall, 319 pp.

Strauch, R. G., D. A. Merritt, K. P. Moran, K. B. Earnshaw, and D. V. de Kamp, 1984: The Colorado wind-profiling network. $J$. Atmos. Oceanic Technol., 1, 37-49.

Weber, B. L., and Coauthors, 1990: Preliminary evaluation of the first NOAA Demonstration Network Wind Profiler. J. Atmos. Oceanic Technol., 7, 909-918.

Woodman, R. F., 1985: Spectral moment estimation in MST radars. Radio Sci., 20, 1185-1195.

Worthington, R. M., 2001: Asymmetry of atmospheric microstructure over synoptic scales. Ann. Geophys., 19, 921-924.

Yu, T.-Y., and R. D. Palmer, 2001: Atmospheric radar imaging using spatial and frequency diversity. Radio Sci., 36, 1493-1504. 